\title{
Functionality, comorbidity, complication \& surgery of hip fracture in older adults by age distribution
}

\author{
Sonia Jiménez-Mola ${ }^{1}$ \\ iD César Calvo-Lobo² \\ Javier Idoate-Gil $\mathbf{1}$ \\ Jesús Seco-Calvo
}

1. Ortogeiatric Unit, Complejo Asistencial Universitario de León (CAULE), León, Spain. 2. Nursing and Physical Therapy Department, Institute of Biomedicine (IBIOMED), University of León, León, Spain. 3. Institute of Biomedicine (IBIOMED), University of León, León, Spain. Visiting Researcher at the University of the Basque Country, Leioa, Basque Country, Spain.

\section{SUMMARY}

BACKGROUND: Hip fractures may be the greatest complication secondary to osteoporotic disorder. The objective of this study was to determine the influence of age distribution in the functionality, comorbidity, complications and surgical features of older adults with hip fractures.

METHODS: A prospective cohort study was carried out from 2013 to 2014. A sample of 557 adults over 75 years old with osteoporotic hip fractures was recruited from the Orthogeriatric Unit of the León University Hospital (Spain). Age distributions of 75-84, 85-90 and $>90$ years old were considered. Firstly, sociodemographic data, fracture type and hospital staying days were collected. Secondly, baseline functionality (Barthel index), ambulation, cognitive impairment and comorbidities were described. Thirdly, surgical intervention, urgency, type, American Association of Anesthesiologists (ASA) scores, non-surgical cause, and baseline pharmacologic treatments were determined. Finally, complications and features at hospital discharge were observed.

RESULTS: The age ranges did not show any statistically-significant differences $\left(P<.05 ; R^{2}=.000-.005\right)$ for gender, fracture type, or number of hospital staying days. Statistically-significant differences $\left(P<.05 ; R^{2}=.011-.247\right)$ between age groups were observed for Barthel index, cognitive impairment, dementia, osteoporosis, Parkinson's disease, aortic stenosis, surgery type, ASA-score, non-surgical cause, benzodiazepines, antidementia, anti-osteoporosis, insulin, pharmacologic treatments, renal function alteration, heart failure, destination and ambulation features. All other measurements did not show statistically-significant differences $\left(P>.05 ; R^{2}=.000-.010\right)$. CONCLUSION: Age distributions greater than 75 years old may determine the functionality, comorbidities, surgical features, baseline pharmacologic treatments, complications and features at hospital discharge for older adults who suffer a hip fracture.

KEYWORDS: Age Distribution. Frail Elderly. Hip Fractures. Musculoskeletal Diseases.

\section{INTRODUCTION}

Worldwide, hip fractures may be considered as the major complication in terms of morbid-mortality and economic burden secondary to the osteoporotic disorder. ${ }^{1}$ Regarding the southern European population, a high prevalence and incidence were observed in Spain, especially regarding trochanteric fractures, female gender and ages over 85 years. ${ }^{2,3}$ the community with the highest incidence of $\mathrm{HF}$ in Spain. Methods data about age, gender, type of frac- ture and month of hospitalisation among patients aged 65 years and older discharged with a diagnosis of HF were collected. Crude and age-standardised annual incidence rate were reckoned. To analyse HF trend, the age/sex-adjusted average annual change in incidence (incidence rate ratio, IRR Furthermore, the relationship between age and mortality after a hip fracture in older adults may reach $5.5 \%$ and can be associated with several comorbidities, such as con-

DATE OF SUBMISSION: 10-Sep-2017

DATE OF ACCEPTANCE: 24-Oct-2017

CORRESPONDING AUTHOR: César Calvo-Lobo

Av. Astorga, s/n - Ponferrada - León

Spain - 24401 - Tel: 605811782 - Fax: 605

fjavieridoate@gmail.com

dr.seco.jesus@gmail.com

E-mail: cecalvo19@hotmail.com soniajimenezmola@hotmail.com 
gestive heart failure, metastasis, fluid and electrolyte disorders, coagulation deficiencies, or liver disease. ${ }^{4}$

Indeed, older adults who suffered hip fractures in a hospital may present a worse surgical (grade III and IV of the American Association of Anesthesiologists - ASA score) and mortality risk than those who suffered hip fractures in the community. ${ }^{5}$ Medical treatments associated to hip fracture can comprise conservative or invasive procedures. ${ }^{6}$ The conservative treatment may produce a high rate of mobility, mortality and local complications. ${ }^{7}$ Whereas, surgical intervention (overall hemiarthroplasty and total hip arthroplasty) may be the first line of treatment in older adults with a hip fracture, since it allows for an earlier mobilization and reduces complications such as respiratory, infection, circulatory or wound conditions. $^{8}$

After a hip fracture, older adults showed the reductions in quality of life and functionality due to associated balance and mobility impairments. Consequently, this suggested that the majority of these older adults did not return to their pre-fracture lifestyle. ${ }^{9}$ Therefore, the objective of this study was to determine the influence of age distribution in the functionality, comorbidity, complications and surgical features of hip fractures in older adults.

\section{METHODS \\ Design}

A prospective cohort study was carried out from December 2013 to November 2014. The Strengthening the Reporting of Observational Studies in Epidemiology (STROBE) guidelines were considered. ${ }^{10}$

\section{Ethical considerations}

The study was approved by the Clinical Research Ethics Committee of the León University (Spain; code ÉTICA-ULE-004-2015). An informed written consent form was obtained from all participants before their inclusion in the research study. Furthermore, the Helsinki Declaration, Protection Data Organic Law (15/1999) and ethical standards in human experimentation were respected.

\section{Sample size}

Based on the hip fracture incidence in Spain, Azagra et al. ${ }^{11}$ showed a similar rate of 517 new hip fractures from 100,000 older adults per year. Considering the assumed $4 \%$ error for a possible loss in follow-up, 534 participants were necessary to justify the sample size.

\section{Participants}

A sample of 557 older adults with hip fracture was recruited from the Traumatology Unit of the León University Hospital, León (Spain). A consecutive sampling method was used to select the participants in the present study.

The inclusion criteria were: adults over 75 years old who suffered an osteoporotic hip fracture from December $1^{\text {st }}$ of 2013, to November 31 of 2014 recruited from the Orthogeriatric Unit of the León University Hospital. ${ }^{1}$

The exclusion criteria were: pathological fractures secondary to other conditions different from osteoporosis (such as neoplasia or osteomyelitis) ${ }^{12}$ traumatic fractures, ${ }^{13}$ treatment type, and inpatient mortality of traumatic hip fractures are important health policy issues. We showed that insurance status and treatment in university hospitals were significantly associated with treatment type (i.e., primary hip replacement or periprosthetic fractures. ${ }^{14}$

\section{Procedure ant outcomes}

All data were extracted from the medical records by the same authorized investigator (SJM). Age distributions of 75-84, 85-90 and > 90 years old were considered. ${ }^{4}$ Firstly, data on sociodemographic (age and gender), fracture type (subcapital or pertrochanteric fractures), total number of hospital staying days and before the surgery were collected. ${ }^{8}$

Secondly, baseline functionality, based on the Barthel index (total, severe, moderate, slight or no dependence), ${ }^{15}$ ambulation (independence/1 stick; walker/2 sticks; high assistance; not walk), ${ }^{16}$ and cognitive impairment (severe, moderate, slight and no impairment), ${ }^{17} 65$ years or older, with a hip fracture. Mobility and Cognitive status were measured by Tinetti Performance-Oriented Mobility Assessment and Pfeiffers' Scale (Short Portable Mental State Questionnaire as well as comorbidities (cardiopathy, hypertension, depression, dementia, diabetes, osteoarthritis, atrial fibrillation, visual impairment, ictus, chronic renal failure, chronic obstructive pulmonary disease, cancer, multiple falls, anemia, osteoporosis, peripheral vascular disease, ischemic heart disease, prior hip fracture, Parkinson's disease, dysphagia and aortic stenosis) were described. $^{4}$ 
Thirdly, surgical features such as surgical intervention, surgical urgency, surgery type (nail, partial bipolar prosthesis, monopolar prosthesis, total prosthesis and screws), ${ }^{8}$ ASA scores (II - Moderate Systemic Disease; III - Severe Non-disabling Systemic Disease; IV - Severe Vital-risk Systemic Disease), ${ }^{18}$ and non-surgical cause (death, orthopedic care, high surgical risk and hospital transfer), ${ }^{5}$ as well as baseline pharmacologic treatments (anti-hypertensives, benzodiazepines, antidepressants, proton-pump inhibitors, antiplatelets, anticoagulants, oral antidiabetic agents, analgesics, antidementia, neuroleptics, anti-osteoporosis, bronchodilators, domiciliary Oxygen, anti-Parkinsonians and Insulin) were determined. ${ }^{19}$

Finally, complications (anemia, transfusion, delirium, constipation, renal function alteration, urinary tract infection, infection or respiratory insufficiency, malnutrition, heart failure, acute retention of urine, ischemic heart disease, death, pressure ulcers, seroma, surgical wound infection, ictus, venous thrombosis or thromboembolism), ${ }^{20}$ and features at hospital discharge (destination, home move, ambulation and discharge) were observed. ${ }^{21}$

\section{STATISTICAL ANALYSIS}

Statistical analysis was performed using the statistical package SPSS 22.0 (IBM SPSS Inc., Chicago. IL, USA). A confidence interval (CI) of $95 \%$ and a $P$-value $<.05$ were considered statistically significant. The sample was divided into 3 age distributions (75-84, 85-90 and $>90$ years old) in order to determine differences between these groups. ${ }^{4}$ Initially, normality analysis were performed by the Kolmogorov-Smirnov test. Then, a descriptive analysis of the data was carried out. For the quantitative variables, the mean and standard deviation (SD) as well as the one-factor analysis of variance (ANOVA) were calculated for the total number of hospital staying days and before surgery. For the rest of qualitative outcomes, percentage and frequency as well as the Chi-square $\left(\chi^{2}\right)$ test were used. The effect size was calculated by the $R^{2}$ coefficient (slight $\sim$.050; moderate .150; high .250; large .360; and very large .450). ${ }^{22}$

\section{RESULTS}

A sample of 557 participants with hip fractures were received during the follow-up. Periprosthetic fractures $(n=19)$ and pathological fractures $(n=4)$ were excluded. From the remaining participants (n = 534), 31 (5.8\%) older adults expired during admission, and $6(1.1 \%)$ were referred to a different hospital for the intervention $(n=497)$. Age distributions of 75$84(\mathrm{n}=189 ; 46$ men and 143 women; 94 subcapital and 95 pertrochanteric fractures), 85-90 $(\mathrm{n}=180 ; 47$ men and 133 women; 78 subcapital and 102 pertrochanteric fractures $)$ and $>90(\mathrm{n}=165 ; 42$ men and 123 women; 68 subcapital and 97 pertrochanteric fractures) did not show any statistically-significant differences for gender $\left(\chi^{2}=0.16 ; P=.924 ; R^{2}=.000\right)$ or fracture type $\left(\chi^{2}=2.87 ; P=.238 ; R^{2}=0.005\right)$, as well as for total of hospital staying days $(\mathrm{F}=0.08$; $P$ $\left.=.921 ; R^{2}=.000\right)$, mean $(\mathrm{SD})$ varied from $11.15(6.12)$ to $11.43(7.84)$ days, and before surgery $(\mathrm{F}=0.32 ; P=$ $\left..726 ; R^{2}=.001\right)$, mean $(\mathrm{SD})$ varied $5.76(3.52)$ from to 6.13 (4.70) days.

Regarding baseline functionality and comorbidities (Table 1), statistically-significant differences between age distributions were observed for Barthel index $\left(\chi^{2}=35.06 ; P<.001 ; R^{2}=.062\right)$, cognitive impairment $\left(\chi^{2}=31.28 ; P=<.001 ; R^{2}=.055\right)$, dementia $\left(\chi^{2}=8.60 ; P=.014 ; R^{2}=.016\right)$, osteoporosis $\left(\chi^{2}=6.07 ;\right.$ $\left.P=.048 ; R^{2}=.011\right)$, Parkinson's disease $\left(\chi^{2}=6.35 ; P=\right.$ $\left..048 ; R^{2}=.012\right)$ and aortic stenosis $\left(\chi^{2}=7.08 ; P=.029\right.$; $\left.R^{2}=.013\right)$. All other measurements did not show any statistically-significant difference $\left(P>.05 ; R^{2}=.000\right.$ $-.007)$.

Considering surgical features and baseline pharmacologic treatments (Table 2), statistically-significant differences between age ranges were found for surgery type $\left(\chi^{2}=88.34 ; P<.001 ; R^{2}=.151\right)$, ASA score $\left(\chi^{2}=12.22 ; P=.016 ; R^{2}=.023\right)$, non-surgical cause $\left(\chi^{2}=14.53 ; P=.024 ; R^{2}=.247\right)$, benzodiazepines $\left(\chi^{2}=13.29 ; P=.001 ; R^{2}=.025\right)$, antidementia $\left(\chi^{2}=\right.$ $\left.6.77 ; P=.034 ; R^{2}=.013\right)$, anti-osteoporosis $\left(\chi^{2}=5.60\right.$; $\left.P=.049 ; R^{2}=.011\right)$ and insulin $\left(\chi^{2}=9.51 ; P=.009 ; R^{2}\right.$ $=.018)$ pharmacologic treatments. The rest of measurements did not show any statistically significant difference $\left(P>.05 ; R^{2}=.000-.010\right)$.

With respect to complications and features at hospital discharge (Table 3), statistically-significant differences between age groups were observed for renal function alteration $\left(\chi^{2}=8.99 ; P=.011 ; R^{2}=.017\right)$, heart failure $\left(\chi^{2}=7.08 ; P=.029 ; R^{2}=.013\right)$, destination $\left(\chi^{2}=\right.$ $\left.19.22 ; P=.004 ; R^{2}=.038\right)$ and ambulation $\left(\chi^{2}=19.14\right.$; $\left.P=.004 ; R^{2}=.037\right)$ features. All other measurements did not show any statistically significant difference $\left(P>.05 ; R^{2}=.001-.009\right)$. 
TABLE 1. BASELINE FUNCTIONALITY AND COMORBIDITIES OF OLDER ADULTS WITH HIP FRACTURE BY AGE DISTRIBUTION.

\begin{tabular}{|c|c|c|c|c|c|c|c|c|}
\hline \multirow[b]{2}{*}{ Outcomes } & \multirow{2}{*}{$\begin{array}{l}\text { Category } \\
(\mathrm{N}=189) \\
75-84\end{array}$} & \multicolumn{3}{|c|}{ Age distribution (y) } & \multirow[b]{2}{*}{$\chi^{2}$} & \multirow[b]{2}{*}{ Df } & \multirow[b]{2}{*}{$\mathrm{P}^{\dagger}$} & \multirow{2}{*}{$\begin{array}{c}\text { Effect } \\
\text { size } \\
\mathrm{R}^{2}\end{array}$} \\
\hline & & $\begin{array}{l}(N=180) \\
85-90\end{array}$ & $\begin{array}{l}(\mathrm{N}=165) \\
>90\end{array}$ & & & & & \\
\hline \multicolumn{9}{|l|}{ Functionality } \\
\hline \multirow[t]{5}{*}{ BARTHEL } & Total dependence & $8.5 \%(16)$ & $15.6 \%(28)$ & $12.1 \%(20)$ & \multirow{5}{*}{35.06} & \multirow{5}{*}{8} & \multirow{5}{*}{$.000^{\star \star}$} & \multirow{5}{*}{.062} \\
\hline & Severe dependence & $16.4 \%(31)$ & $22.8 \%(41)$ & $17.6 \%(29)$ & & & & \\
\hline & Moderate dependence & $26.5 \%(50)$ & $26.1 \%(47)$ & $37.6 \%(62)$ & & & & \\
\hline & Slight dependence & $3.7 \%(7)$ & $6.1 \%(11)$ & $11.5 \%(19)$ & & & & \\
\hline & Independence & $45.0 \%(85)$ & $29.4 \%(53)$ & $21.1 \%(35)$ & & & & \\
\hline \multirow[t]{4}{*}{ Ambulation } & Independence/1 stick & $68.8 \%(130)$ & $62.2 \%(112)$ & $61.8 \%(102)$ & \multirow{4}{*}{5.76} & \multirow{4}{*}{6} & \multirow{4}{*}{$.451^{\mathrm{NS}}$} & \multirow{4}{*}{.011} \\
\hline & Walker/2 sticks & $24.3 \%(46)$ & $25.6 \%(46)$ & $28.5 \%(47)$ & & & & \\
\hline & High assistance & $3.7 \%(7)$ & $7.2 \%(13)$ & $7.3 \%(12)$ & & & & \\
\hline & Not walk & $3.2 \%(6)$ & $5.0 \%(9)$ & $2.4 \%(4)$ & & & & \\
\hline \multirow[t]{4}{*}{ Cognitive impairment } & No impairment & $70.4 \%(133)$ & $43.9 \%(79)$ & $49.1 \%(81)$ & \multirow{4}{*}{31.28} & \multirow{4}{*}{6} & \multirow{4}{*}{$.000^{\star \star}$} & \multirow{4}{*}{.055} \\
\hline & Slight & $11.6 \%(22)$ & $25.0 \%(45)$ & $25.5 \%(42)$ & & & & \\
\hline & Moderate & $14.8 \%(28)$ & $25.0 \%(45)$ & $21.8 \%(36)$ & & & & \\
\hline & Severe & $3.2 \%(6)$ & $6.1 \%(11)$ & $3.6 \%(6)$ & & & & \\
\hline \multicolumn{9}{|l|}{ Comorbidities } \\
\hline Cardiopathy & 400 & $72.5 \%(137)$ & $77.2 \%(139)$ & $75.2 \%(124)$ & 1.11 & 2 & $.575^{N S}$ & .002 \\
\hline Hypertension & 377 & $67.7 \%(128)$ & $71.1 \%(128)$ & $73.3 \%(121)$ & 1.37 & 2 & $.504^{\mathrm{NS}}$ & .003 \\
\hline Depression & 158 & $29.1 \%(55)$ & $31.7 \%(57)$ & $27.9 \%(46)$ & 0.63 & 2 & $.731^{\mathrm{NS}}$ & .001 \\
\hline Dementia & 132 & $18.0 \%(34)$ & $31.1 \%(56)$ & $25.5 \%(42)$ & 8.60 & 2 & $.014 *$ & .016 \\
\hline Diabetes & 127 & $27.5 \%(52)$ & $24.4 \%(44)$ & $18.8 \%(31)$ & 3.77 & 2 & $.152^{N S}$ & .007 \\
\hline Osteoarthritis & 117 & $24.9 \%(47)$ & $20.6 \%(37)$ & $20.0 \%(33)$ & 1.51 & 2 & .470 NS & .003 \\
\hline Atrial fibrillation & 107 & $21.2 \%(40)$ & $21.1 \%(38)$ & $17.6 \%(29)$ & 0.90 & 2 & $.637^{\mathrm{NS}}$ & .002 \\
\hline Visual impairment & 89 & $13.8 \%(26)$ & $18.3 \%(33)$ & $18.2 \%(30)$ & 1.79 & 2 & .410 NS & .003 \\
\hline Ictus & 85 & $16.4 \%(31)$ & $15.6 \%(28)$ & $15.8 \%(26)$ & 0.05 & 2 & .973 NS & .000 \\
\hline Chronic renal failure & 83 & $13.2 \%(25)$ & $18.3 \%(33)$ & $15.2 \%(25)$ & 1.86 & 2 & .395 NS & .003 \\
\hline COPD & 82 & $18.0 \%(34)$ & $16.7 \%(30)$ & $10.9 \%(18)$ & 3.76 & 2 & $.153^{N S}$ & .007 \\
\hline Cancer & 72 & $13.2 \%(25)$ & $11.7 \%(21)$ & $15.8 \%(26)$ & 1.25 & 2 & $.535^{N S}$ & .002 \\
\hline Multiple falls & 62 & $13.2 \%(25)$ & $11.7 \%(21)$ & $9.7 \%(16)$ & 1.07 & 2 & $.585^{N S}$ & .002 \\
\hline Anemia & 61 & $10.1 \%(19)$ & $10.0 \%(18)$ & $14.5 \%(24)$ & 2.30 & 2 & $.317^{\mathrm{NS}}$ & .004 \\
\hline Osteoporosis & 58 & $14.8 \%(28)$ & $10.6 \%(19)$ & $6.7 \%(11)$ & 6.07 & 2 & $.048^{*}$ & .011 \\
\hline $\begin{array}{l}\text { Peripheral vascular } \\
\text { disease }\end{array}$ & 56 & $11.1 \%(21)$ & $7.8 \%(14)$ & $12.7 \%(21)$ & 2.37 & 2 & .306 NS & .004 \\
\hline Ischemic heart disease & 46 & $9.5 \%(18)$ & $8.9 \%(16)$ & $7.3 \%(12)$ & 0.59 & 2 & .743 NS & .001 \\
\hline Prior hip fracture & 38 & $6.3 \%(12)$ & $7.2 \%(13)$ & $7.9 \%(13)$ & 0.32 & 2 & .854 NS & .001 \\
\hline Parkinson's disease & 28 & $5.8 \%(11)$ & $7.8 \%(14)$ & $1.8 \%(3)$ & 6.35 & 2 & $.042^{*}$ & .012 \\
\hline Dysphagia & 17 & $2.1 \%(4)$ & $3.9 \%(7)$ & $3.6 \%(6)$ & 1.10 & 2 & $.577^{N S}$ & .002 \\
\hline Aortic stenosis & 13 & $4.8 \%(9)$ & $1.7 \%(3)$ & $0.6 \%(1)$ & 7.08 & 2 & $.029^{*}$ & .013 \\
\hline
\end{tabular}

Ns $=$ Non statistically significant different with $P>$.05. ${ }^{*}$ Statistically significant differences with $P<0.05 .{ }^{* \star}=$ Statistically significant differences with $P<0.01 .{ }^{\dagger}=$ Chi square test $\left(\chi^{2}\right)$ was applied, Bold numbers determine the most significant contribution. Abbreviations: COPD, chronic obstructive pulmonary disease; Df, degrees of freedom.

\section{DISCUSSION}

The present study supports novel evidence on functionality, comorbidity, complications and surgical features of hip fractures in older adults over 75 years old by age distribution. It determines the key points to consider in the aging process of older adults during and after hip fracture. All sociodemographic data, fracture type and hospital staying days were representative of the general population of Spain and in accordance to prior studies. .11,23,24 $^{2}$

Considering baseline functionality and comorbidities (Table 1), there were only slight effects observed for the Barthel index, cognitive impairment, dementia, osteoporosis, Parkinson's disease and aortic stenosis. Therefore, independence, osteoporosis, aortic 
TABLE 2. SURGICAL FEATURES AND BASELINE PHARMACOLOGIC TREATMENTS OF OLDER ADULTS WITH HIP FRACTURE BY AGE DISTRIBUTION.

\begin{tabular}{|c|c|c|c|c|c|c|c|c|}
\hline \multirow{2}{*}{$\begin{array}{l}\text { Outcomes } \\
75-84\end{array}$} & \multirow{2}{*}{$\begin{array}{l}\text { Category / n } \\
85-90\end{array}$} & \multicolumn{3}{|c|}{ Age distribution (y) } & \multirow{2}{*}{$\chi^{2}$} & \multirow[t]{2}{*}{ Df } & \multirow[t]{2}{*}{$\mathrm{P}^{\dagger}$} & \multirow{2}{*}{$\begin{array}{l}\text { Effect } \\
\text { size } R^{2}\end{array}$} \\
\hline & & $>90$ & & & & & & \\
\hline \multicolumn{9}{|l|}{ Surgical characteristics } \\
\hline Surgery & Sí & $92.6 \%(175)$ & 96.1\% (173) & $91.5 \%(151)$ & 3.32 & 2 & .190 NS & .006 \\
\hline Surgical urgency & Sí & $12.7 \%(24)$ & $14.4 \%(26)$ & $12.1 \%(20)$ & 0.45 & 2 & .798 NS & .001 \\
\hline \multirow[t]{5}{*}{ Surgery type } & Nail & $49.7 \%(87)$ & $56.5 \%(98)$ & $59.6 \%(90)$ & \multirow{5}{*}{88.34} & \multirow{5}{*}{8} & \multirow{5}{*}{$.000^{\star *}$} & \multirow{5}{*}{.151} \\
\hline & Partial bipolar prosthesis & $28.6 \%(50)$ & $34.1 \%(59)$ & $19.9 \%(30)$ & & & & \\
\hline & Monopolar prosthesis & $1.1 \%(2)$ & $5.2 \%(9)$ & $17.9 \%(27)$ & & & & \\
\hline & Total prosthesis & $16.0 \%(28)$ & $0.6 \%(1)$ & -- & & & & \\
\hline & Screws & $4.6 \%(8)$ & $3.5 \%(6)$ & $2.6 \%(4)$ & & & & \\
\hline \multirow[t]{3}{*}{ ASA scores } & II - Grade & $31.9 \%(60)$ & $21.7 \%(39)$ & $18.2 \%(30)$ & \multirow{3}{*}{12.22} & \multirow{3}{*}{4} & \multirow{3}{*}{$.016^{*}$} & \multirow{3}{*}{.023} \\
\hline & III - Grade & $53.7 \%(101)$ & $67.2 \%(121)$ & $68.5 \%(113)$ & & & & \\
\hline & IV - Grade & $14.4 \%(27)$ & $11.1 \%(20)$ & $13.3 \%(22)$ & & & & \\
\hline Non-surgical & Death $(n=15)$ & $28.6 \%(4)$ & $42.9 \%(3)$ & $57.1 \%(8)$ & \multirow{4}{*}{14.53} & \multirow{4}{*}{6} & \multirow{4}{*}{$.024^{*}$} & \multirow{4}{*}{.247} \\
\hline \multirow[t]{3}{*}{ cause } & Orthopedic care $(n=7)$ & $7.1 \%(1)$ & $42.9 \%(3)$ & $21.5 \%(3)$ & & & & \\
\hline & High surgical risk $(n=7)$ & $28.6 \%(4)$ & $0 \%$ & $21.4 \%(3)$ & & & & \\
\hline & Hospital transfer $(n=6)$ & $35.7 \% \quad(n=5)$ & $14.3 \%(n=1)$ & $0 \%$ & & & & \\
\hline \multicolumn{9}{|c|}{ Pharmacologic treatments at baseline } \\
\hline Anti-hypertensives & 390 & $68.3 \%(129)$ & $75.6 \%(136)$ & $75.8 \%(125)$ & 3.40 & 2 & $.183^{\text {NS }}$ & .006 \\
\hline Benzodiazepines & 189 & $30.7 \%(58)$ & $30.0 \%(54)$ & $46.7 \%(77)$ & 13.29 & 2 & $.001^{\star \star}$ & .025 \\
\hline Antidepressants & 171 & $32.8 \%(62)$ & $35.6 \%(64)$ & $27.3 \%(45)$ & 2.80 & 2 & $.247^{N S}$ & .005 \\
\hline Proton-pump inhibitors & 157 & $28.0 \%(53)$ & $30.6 \%(55)$ & $29.7 \%(49)$ & 0.29 & 2 & $.865^{\text {NS }}$ & .001 \\
\hline Antiplatelets & 150 & $24.3 \%(46)$ & $33.9 \%(61)$ & $26.1 \%(43)$ & 4.65 & 2 & .098 NS & .009 \\
\hline Anticoagulants & 100 & $20.1 \%(38)$ & $21.1 \%(38)$ & $14.5 \%(24)$ & 2.80 & 2 & .246 NS & .005 \\
\hline Oral antidiabetic agents & 96 & $21.2 \%(40)$ & $15.6 \%(28)$ & $17.0 \%(28)$ & 2.13 & 2 & $.345^{\text {NS }}$ & .004 \\
\hline Analgesics & 94 & $22.2 \%(42)$ & $15.0 \%(27)$ & $15.2 \%(25)$ & 4.31 & 2 & .116 NS & .008 \\
\hline Antidementia & 63 & $12.7 \%(24)$ & $15.6 \%(28)$ & $6.7 \%(11)$ & 6.77 & 2 & $.034^{*}$ & .013 \\
\hline Neuroleptics & 52 & $8.5 \%(16)$ & $11.7 \%(21)$ & $9.1 \%(15)$ & 1.19 & 2 & .552 NS & .002 \\
\hline Anti-osteoporosis & 44 & $12.2 \%(23)$ & $6.1 \%(11)$ & $6.1 \%(10)$ & 5.60 & 2 & $.049^{*}$ & .011 \\
\hline Bronchodilators & 40 & $7.9 \%(15)$ & $7.8 \%(14)$ & $6.7 \%(11)$ & 0.24 & 2 & $.888^{N S}$ & .000 \\
\hline Domiciliary Oxygen & 28 & $4.8 \%(9)$ & $6.7 \%(12)$ & $4.2 \%(7)$ & 1.16 & 2 & $.561^{\text {NS }}$ & .002 \\
\hline Anti-Parkinsonians & 28 & $6.3 \%(12)$ & $7.2 \%(13)$ & $1.8 \%(3)$ & 5.78 & 2 & $.056^{\text {NS }}$ & .010 \\
\hline Insulin & 24 & $7.9 \%(15)$ & $3.9 \%(7)$ & $1.2 \%(2)$ & 9.51 & 2 & $.009^{\star *}$ & .018 \\
\hline
\end{tabular}

${ }^{\text {NS }}=$ Non statistically significant different with $P>05$. ${ }^{*}$ Statistically significant differences with $P<0.05 .{ }^{* *}=$ Statistically significant differences with $P<0.01 .{ }^{\dagger}=$ Chi square test $\left(\chi^{2}\right)$ was applied, Bold numbers determine the most significant contribution. Abbreviations: ASA, American Association of Anesthesiologists - ASA scores (II - Moderate Systemic Disease; III - Severe Non-disabling Systemic Disease; IV - Severe Vital-risk Systemic Disease); Df, degrees of freedom.

stenosis and Parkinson's disease may be more frequent in younger age distributions of older adults, while cognitive impairment and dementia seemed to be presented in older adults over 85 years old, coinciding with previous researches. ${ }^{4,15,17}$ Furthermore, Parkinson's disease may decrease in the older elderly patients, since it may be considered a neurodegenerative condition with associated life expectative reduction. ${ }^{25}$

Regarding surgical features and baseline pharmacologic treatments (Table 2), high effects were observed for surgery type, non-surgical cause, and insulin pharmacologic treatment, while slight effects were determined for ASA score, benzodiazepines, antidementia and anti-osteoporosis drugs. Partial bipolar prosthesis and monopolar prosthesis seemed to be more common in older adults over $90 .^{8}$ Moreover, the IV - ASA score did not seem to variate with age distribution. Nevertheless, the II and III - ASA scores may be associated with 75-84 and 85-90 age groups, respectively. Therefore, increased age is not associated with higher surgical risk in geriatric hip fracture patients. ${ }^{24}$ Anti-dementia and benzodiazepines may be more frequent in nonagenarian patients, while anti-osteoporosis and insulin seemed to be more common in the 75-84 age group. In this 
TABLE 3. COMPLICATIONS AND FEATURES AT HOSPITAL DISCHARGE OF OLDER ADULTS WITH HIP FRACTURE BY AGE DISTRIBUTION.

\begin{tabular}{|c|c|c|c|c|c|c|c|c|}
\hline \multirow[t]{2}{*}{ Outcomes } & \multirow{2}{*}{$\begin{array}{l}N \\
(N=189) \\
75-84\end{array}$} & \multicolumn{3}{|c|}{ Age distribution (y) } & \multirow[t]{2}{*}{$\chi^{2}$} & \multirow[t]{2}{*}{ Df } & \multirow[t]{2}{*}{$\mathrm{P}^{+}$} & \multirow{2}{*}{$\begin{array}{l}\text { Effect } \\
\text { size } \mathrm{R}^{2}\end{array}$} \\
\hline & & $\begin{array}{l}(N=180) \\
85-90\end{array}$ & $\begin{array}{l}(N=165) \\
>90\end{array}$ & & & & & \\
\hline \multicolumn{9}{|l|}{ Complicaciones } \\
\hline Anemia & 469 & $88.4 \%(167)$ & $85.0 \%(153)$ & $90.3 \%(149)$ & 2.34 & 2 & $.310^{\text {NS }}$ & .004 \\
\hline Transfusion & 208 & $36.5 \%(69)$ & $40.0 \%(72)$ & $40.6 \%(67)$ & 0.75 & 2 & $.688^{\mathrm{NS}}$ & .001 \\
\hline Delirium & 196 & $30.7 \%(58)$ & $38.9 \%(70)$ & $41.2 \%(68)$ & 4.76 & 2 & .093 NS & .009 \\
\hline Constipation & 117 & $24.9 \%(47)$ & $17.2 \%(31)$ & $23.6 \%(39)$ & 3.57 & 2 & .168 NS & .007 \\
\hline Renal function alteration & 94 & $13.2 \%(25)$ & $15.6 \%(28)$ & $24.8 \%(41)$ & 8.99 & 2 & $.011^{*}$ & .017 \\
\hline Urinary tract infection & 81 & $12.7 \%(24)$ & $16.1 \%(29)$ & $17.0 \%(28)$ & 1.44 & 2 & .488 NS & .003 \\
\hline Infection / Respiratory insufficiency & 79 & $13.2 \%(25)$ & $13.3 \%(24)$ & $18.2 \%(30)$ & 2.18 & 2 & $.337^{N S}$ & .004 \\
\hline Malnutrition & 74 & $14.8 \%(28)$ & $12.2 \%(22)$ & $14.5 \%(24)$ & 0.61 & 2 & .736 NS & .001 \\
\hline Heart failure & 64 & $9.5 \%(18)$ & $9.4 \%(17)$ & $17.6 \%(29)$ & 7.08 & 2 & $.029 *$ & .013 \\
\hline Acute retention of urine & 50 & $8.5 \%(16)$ & $10.0 \%(18)$ & $9.7 \%(16)$ & 0.29 & 2 & $.866^{\mathrm{NS}}$ & .001 \\
\hline Ischemic heart disease & 39 & $9.5 \%(18)$ & $7.8 \%(14)$ & $4.2 \%(7)$ & 3.72 & 2 & .156 NS & .007 \\
\hline Death & 31 & $4.8 \%(9)$ & $3.9 \%(7)$ & $9.1 \%(15)$ & 4.84 & 2 & $.089^{N S}$ & .009 \\
\hline Pressure ulcers & 21 & $5.3 \%(10)$ & $2.2 \%(4)$ & $4.2 \%(7)$ & 2.36 & 2 & $.307^{N S}$ & .004 \\
\hline Seroma & 9 & $2.6 \%(5)$ & $0.6 \%(1)$ & $1.8 \%(3)$ & -- & -- & -- & -- \\
\hline Surgical wound infection & 4 & $1.1 \%(2)$ & $0.6 \%(1)$ & $0.6 \%(1)$ & -- & -- & -- & -- \\
\hline Ictus & 3 & $1.1 \%(2)$ & $0 \%$ & $0.6 \%(1)$ & -- & -- & -- & -- \\
\hline $\begin{array}{l}\text { Venous thrombosis/Thromboem- } \\
\text { bolism }\end{array}$ & 2 & $0 \%$ & $0 \%$ & $1.2 \%(2)$ & -- & -- & -- & -- \\
\hline \multicolumn{9}{|l|}{ Features at hospital discharge } \\
\hline \multirow[t]{4}{*}{ Destination } & $\begin{array}{l}\text { Concerted care } \\
\text { center }\end{array}$ & $31.6 \%(55)$ & $27.6 \%(47)$ & $37.3 \%(56)$ & \multirow{4}{*}{19.22} & \multirow{4}{*}{6} & \multirow{4}{*}{$.004^{* *}$} & \multirow{4}{*}{.038} \\
\hline & Nursing home & $25.3 \%(44)$ & $33.5 \%(57)$ & $35.3 \%(53)$ & & & & \\
\hline & Family home & $17.2 \%(30)$ & $22.9 \%(39)$ & $17.3 \%(26)$ & & & & \\
\hline & Own home & $25.9 \%(45)$ & $15.9 \%(27)$ & $10.0 \%(15)$ & & & & \\
\hline \multirow[t]{3}{*}{ Home move } & Yes & $10.3 \%(18)$ & $11.1 \%(19)$ & $8.0 \%(12)$ & \multirow{3}{*}{4.35} & \multirow{3}{*}{4} & \multirow{3}{*}{$.361^{\mathrm{NS}}$} & \multirow{3}{*}{.009} \\
\hline & No & $58.6 \%(102)$ & $62.0 \%(106)$ & $54.7 \%(82)$ & & & & \\
\hline & $\begin{array}{l}\text { Hospital con- } \\
\text { certed center }\end{array}$ & $31.0 \%(54)$ & $26.9 \%(46)$ & $37.3 \%(56)$ & & & & \\
\hline \multirow[t]{4}{*}{ Ambulation } & $\begin{array}{l}\text { Independence/1 } \\
\text { stick }\end{array}$ & $1.7 \%(3)$ & $0 \%$ & $0 \%$ & \multirow{4}{*}{19.14} & \multirow{4}{*}{6} & \multirow{4}{*}{$.004^{\star \star}$} & \multirow{4}{*}{.037} \\
\hline & Walker/2 sticks & $41.1 \%(72)$ & $32.0 \%(55)$ & $22.7 \%(34)$ & & & & \\
\hline & High assistance & $22.9 \%(40)$ & $25.6 \%(44)$ & $29.3 \%(44)$ & & & & \\
\hline & Not walk & $34.3 \%(60)$ & $42.4 \%(73)$ & $48.0 \%(72)$ & & & & \\
\hline Discharge & Yes & $14.9 \%(26)$ & $9.3 \%(16)$ & $16.0 \%(24)$ & 3.71 & 2 & $.157^{N S}$ & .007 \\
\hline
\end{tabular}

sense, some authors have claimed a higher risk of hip fracture in older adults with hypnotic pharmacologic treatment. ${ }^{26}$

Finally, complications and features at hospital discharge (Table 3) showed only slight effects for renal function alteration, heart failure, destination and ambulation features. Coinciding with this, other authors reported kidney function alteration as a frequent complication in older adults with hip fracture. ${ }^{27}$ Additionally, ambulation and lifestyle characteristics may be more dependent in the nonagenarian patients as it was reported by previous authors. ${ }^{28}$

Some limitations should be considered in the present study; despite the age distributions over 75 years old seemed to show the greatest prevalence of hip fracture and presented a similar number among participants in our study, the considered age ranges were not equal. ${ }^{1}$ Indeed, the follow-up after hospital discharge was not carried out. Therefore, rehabilitation, complications and pharmacologic treatments 
after hospital discharge were not collected. New cohort studies may be necessary in order to describe the status of older adults with hip fracture during the rehabilitation phase. ${ }^{29}$ Furthermore, this study did not consider the presence of musculoskeletal alterations in the lower limb such as the myofascial pain syndrome associated to hip fracture. ${ }^{30}$ Further interventional studies may be necessary to improve the clinical features of older adults with muscle conditions associated to hip fracture, according to prior studies in other body regions. ${ }^{31}$

In conclusion, age distributions over 75 years old may determine the functionality, comorbidities, surgical features, baseline pharmacologic treatments, complications and features at hospital discharge for older adults who suffer a hip fracture.

\section{RESUMO}

CONTEXTO:As fraturas do quadril podem ser a maior complicação secundária à doença osteoporótica. O objetivo deste estudo foi determinar a influência da distribuição etária na funcionalidade, comorbidade, complicações e características cirúrgicas de idosos com fratura de quadril.

MÉTODOS: Um estudo prospectivo de coorte foi realizado de 2013-2014. Uma amostra de 557 adultos mais velhos, com mais de 75 anos, com fratura de quadril osteoporótica foi recrutada na Unidade Ortogeriátrica do Hospital Universitário de León (Espanha). As distribuições de idade de 75-84, 85-90 e >90 anos foram consideradas. Em primeiro lugar, foram coletados dados sociodemográficos, tipo de fratura e dias de permanência hospitalar. Em segundo lugar, foram descritas funcionalidades de base (índice Barthel), ambulação, comprometimento cognitivo e comorbidades. Em terceiro lugar, determinaram-se a intervenção cirúrgica, a urgência, o tipo, os resultados da Associação Americana de Anestesiologistas (ASA), a causa não cirúrgica e os tratamentos farmacológicos iniciais. Finalmente, foram observadas complicações e características na alta hospitalar.

RESULTADOS: As faixas etárias não mostraram diferenças estatisticamente significativas $\left(P<, 05 ; R^{2}=, 000-, 005\right)$ para sexo, tipo de fratura ou dias de permanência hospitalar. Foram apresentadas diferenças estatisticamente significativas $\left(P<, 05 ; R^{2}=, 011-, 247\right)$ para o índice de Barthel, comprometimento cognitivo, demência, osteoporose, doença de Parkinson, estenose aórtica, tipo de cirurgia, pontuação ASA, causa não cirúrgica, benzodiazepínicos, antidementia, antiosteoporose, insulina, tratamentos farmacológicos, alteração da função renal, insuficiência cardíaca, destino e características de ambulação entre grupos etários. O restante das medidas não apresentou diferença estatisticamente significativa $\left(P>0,05 ; R^{2}=, 000-, 010\right)$.

CONCLUSÃO: As distribuições de idade após 75 anos podem determinar a funcionalidade, comorbidades, características cirúrgicas, tratamentos farmacológicos de base, complicações e características na alta hospitalar de adultos mais velhos que sofrem fratura de quadril.

PALAVRAS-CHAVE: Distribuição por idade. Idosos fragilizados. Fraturas do quadril. Doenças musculoesqueléticas.

\section{REFERENCES}

1. Boschitsch EP, Durchschlag E, Dimai HP. Age-related prevalence of osteoporosis and fragility fractures: real-world data from an Austrian Menopause and Osteoporosis Clinic. Climacteric. 2017;20(2):157-63.

2. Pueyo-Sánchez MJ, Larrosa M, Suris X, Casado E, Auleda J, Fusté J, et al. Secular trend in the incidence of hip fracture in Catalonia, Spain, 20032014. Age Ageing. 2016;46(2):324-8.

3. Lobo E, Marcos G, Santabárbara |, Salvador-Rosés H, Lobo-Escolar L, De la Cámara C, et al. Gender differences in the incidence of and risk factors for hip fracture: a 16-year longitudinal study in a Southern European population. Maturitas. 2017;97:38-43.

4. Padrón-Monedero A López-Cuadrado T, Galán I, Martínez-Sánchez EV, Martin P, Fernández-Cuenca R. Effect of comorbidities on the association between age and hospital mortality after fall-related hip fracture in elderly patients. Osteoporos Int. 2017;28(5):1559-68.

5. Shabani F, Farrier Al, Smith R, Venkatesan M, Thomas C, Uzoigwe CE, et al. Hip fractures sustained in hospital: comorbidities and outcome. Postgrad Med J. 2015;91(1072):61-4.

6. Li K, Zheng Y. Internal fixation versus conservative treatment for elderly patients with a trochanteric hip fracture in conjunction with post-stroke hemiplegia. Injury. 2016;47(10):2169-72.
7. Kawaji H, Uematsu T, Oba R, Takai S. Conservative treatment for fracture of the proximal femur with complications. I Nippon Med Sch. 2016;83(1):2-5.

8. Mosseri J, Trinquart L, Nizard R, Ravaud P. Meta-analysis of a complex network of non-pharmacological interventions: the example of femoral neck fracture. PLoS One. 2016;11(1):e0146336.

9. Hall SE, Williams |A, Senior |A, Goldswain PR, Criddle RA. Hip fracture outcomes: quality of life and functional status in older adults living in the community. Aust N Z J Med. 2000;30(3):327-32.

10. Vandenbroucke JP, von Elm E, Altman DG, Gøtzsche PC, Mulrow CD, Pocock SI, et al. Strengthening the Reporting of Observational Studies in Epidemiology (STROBE): explanation and elaboration. Int J Surg. 2014;12(12):1500-24.

11. Azagra R, López-Expósito F, Martin-Sánchez JC, Aguyé A, Moreno N, Cooper $C$, et al. Changing trends in the epidemiology of hip fracture in Spain. Osteoporos Int. 2014;25(4):1267-74.

12. Cornelis FH, Tselikas L, Carteret T, Lapuyade B, De Baere T, Le Huec |C, et al. Percutaneous internal fixation with Y-STRUT ${ }^{\circledR}$ device to prevent both osteoporotic and pathological hip fractures: a prospective pilot study. | Orthop Surg Res. 2017;12(1):27. 
13. Mehra T, Moos RM, Seifert B, Bopp M, Senn O, Simmen HP, et al. Impact of structural and economic factors on hospitalization costs, inpatient mortality, and treatment type of traumatic hip fractures in Switzerland. Arch Osteoporos. 2017;12(1):7.

14. Pavelka T, Salášek M, Weisová D. Periprosthetic femoral fractures after total hip replacement: our results and treatment complications. Acta Chir Orthop Traumatol Cech. 2017;84(1):52-8.

15. González-Zabaleta J, Pita-Fernandez S, Seoane-Pillado T, López-Calviño B, Gonzalez-Zabaleta |L. Dependence for basic and instrumental activities of daily living after hip fractures. Arch Gerontol Geriatr. 2015;60(1):66-70.

16. Peiris C, Shields N, Kingsley M, Yeung J, Hau R, Taylor N. Maximum tolerated dose of walking for community-dwelling people recovering from hip fracture: a dose-response trial. Arch Phys Med Rehabil. 2017;98(12):2533-9.

17. Ariza-Vega $P$, Lozano-Lozano M, Olmedo-Requena R, Martín-Martín L, Jiménez-Moleón JJ. Influence of cognitive impairment on mobility recovery of patients with hip fracture. Am J Phys Med Rehabil. 2017;96(2):109-15.

18. Kastanis G, Topalidou A, Alpantaki K, Rosiadis M, Balalis K. Is the ASA score in geriatric hip fractures a predictive factor for complications and readmission? Scientifica (Cairo). 2016;2016:7096245.

19. Lönnbro J, Wallerstedt SM. Clinical relevance of the STOPP/START criteria in hip fracture patients. Eur J Clin Pharmacol. 2017;73(4):499-505.

20. Kua J, Ramason R, Rajamoney G, Chong MS. Which frailty measure is a good predictor of early post-operative complications in elderly hip fracture patients? Arch Orthop Trauma Surg. 2016;136(5):639-47.

21. Regenbogen SE, Cain-Nielsen AH, Norton EC, Chen LM, Birkmeyer JD, Skinner JS. Costs and consequences of early hospital discharge after major inpatient surgery in older adults. JAMA Surg. 2017;152(5):e170123.

22. Preacher KJ, Kelley K. Effect size measures for mediation models: quantitative strategies for communicating indirect effects. Psychol Methods. 2011;16(2):93-115.

23. Tanner DA, Kloseck M, Crilly RG, Chesworth B, Gilliland J. Hip fracture types in men and women change differently with age. BMC Geriatr. 2010;10:12.

24. Eschbach DA, Oberkircher L, Bliemel C, Mohr J, Ruchholtz S, Buecking $B$. Increased age is not associated with higher incidence of complications, longer stay in acute care hospital and in hospital mortality in geriatric hip fracture patients. Maturitas. 2013;74(2):185-9.

25. Ishihara LS, Cheesbrough A, Brayne C, Schrag A. Estimated life expectancy of Parkinson's patients compared with the UK population. | Neurol Neurosurg Psychiatry. 2007;78(12):1304-9.

26. Bakken MS, Engeland A, Engesaeter LB, Ranhoff AH, Hunskaar S, Ruths S. Risk of hip fracture among older people using anxiolytic and hypnotic drugs: a nationwide prospective cohort study. Eur | Clin Pharmacol. 2014;70(7):873-80.

27. Macheras GA, Kateros K, Koutsostathis SD, Papadakis SA, Tsiridis E. Which patients are at risk for kidney dysfunction after hip fracture surgery? Clin Orthop Relat Res. 2013;471(12):3795-802.

28. Leur K, Vroemen JP, Vos DI, Elmans L, van der Laan L. Outcome after osteosynthesis of hip fractures in nonagenarians. Clin Interv Aging. 2014:9:41-9.

29. Thingstad P, Taraldsen K, Saltvedt I, Sletvold O, Vereijken B, Lamb SE, et al. The long-term effect of comprehensive geriatric care on gait after hip fracture: the Trondheim Hip Fracture Trial: a randomised controlled trial. Osteoporos Int. 2016;27(3):933-42.

30. Sanz DR, Lobo CC, López DL, Morales CR, Marín CS, Corbalán IS. Interrater reliability in the clinical evaluation of myofascial trigger points in three ankle muscles. J Manipulative Physiol Ther. 2016;39(9):623-34.

31. Calvo-Lobo C, Pacheco-da-Costa S, Martínez-Martínez J, Rodríguez-Sanz D, Cuesta-Álvaro P, López-López D. Dry needling on the infraspinatus latent and active myofascial trigger points in older adults with nonspecific shoulder pain: a randomized clinical trial. | Geriatr Phys Ther. 2016:41(1):1-13. 